\title{
Diffractive chains of plasmonic nanolenses: combining near-field focusing and collective enhancement mechanisms
}

\author{
E. Almpanis, ${ }^{1, *}$ N. Papanikolaou, ${ }^{1}$ B. Auguié, ${ }^{2}$ C. Tserkezis,${ }^{3,4}$ and N. Stefanou ${ }^{4}$ \\ ${ }^{1}$ Department of Microelectronics, Institute of Advanced Materials, Physicochemical Processes, \\ Nanotechnology and Microsystems, NCSR “Demokritos," Athens GR-153 10, Greece \\ ${ }^{2}$ The MacDiarmid Institute for Advanced Materials and Nanotechnology, School of Chemical and Physical Sciences, \\ Victoria University of Wellington, P.O. Box 600, Wellington 6140, New Zealand \\ ${ }^{3}$ Donostia International Physics Center (DIPC) and Centro de Física de Materiales CSIC-UPV/EHU, \\ Paseo Manuel de Lardizabal 5, Donostia-San Sebastián 20018, Spain \\ ${ }^{4}$ Section of Solid State Physics, University of Athens, Panepistimioupolis, Athens GR-157 84, Greece \\ *Corresponding author: almpanis@imel.demokritos.gr \\ Received July 12, 2012; revised September 21, 2012; accepted September 28, 2012; \\ posted October 1, 2012 (Doc. ID 172494); published November 6, 2012

\begin{abstract}
We study, by means of full-electrodynamic calculations using the layer-multiple-scattering method, the effect of diffractive coupling on the enhancement of the local electromagnetic field in periodic arrays of nanolenses consisting of three silver spheres with progressively decreasing sizes and separations. The interaction between the hot-spot modes of an isolated nanolens with the Rayleigh-Wood anomalies of the periodic lattice leads to a further enhancement of the local field intensity, which can be controlled by an appropriate choice of the geometrical parameters involved. (C) 2012 Optical Society of America
\end{abstract} \\ OCIS codes: $\quad 050.1965,250.5403,050.5298$.
}

Plasmonic nanoantennas and nanolenses, consisting of two or more metallic nanoparticles in proximity, have recently attracted much interest due to their ability to produce highly confined electromagnetic (EM) fields in the interparticle region, thus leading to enhanced Raman scattering, fluorescence, absorption [1,2], and nonlinear effects [3]. Among the various designs that have been proposed so far, of particular interest are nanolenses consisting of self-similar chains of metallic nanospheres with decreasing radii and separations. It has been shown that, when such a nanolens is optically excited, a hotspot, where the local EM field is enhanced by orders of magnitude due to a multiplicative cascade effect, is formed in the region between the two smallest spheres $[\underline{4}, \underline{5}]$. This local field enhancement in fractal nanoparticle aggregates is correlated with a local anisotropy factor [ $[6]$, while the underlying mechanism can be elucidated in the light of a simple coupled-dipole model [7]. Such chains have been recently fabricated in the laboratory by use of DNA templates [8].

From a different perspective, and indeed with a different mechanism, it is well established that strong enhancement of the local EM field can also occur in periodic structures of metallic nanoparticles [9]. When plasmonic nanoparticles are arranged in sparse periodic lattices, the localized resonance of each particle can be strongly affected by a long-range interaction with the other particles through a coherent multiple-scattering process in the plane of the array. In this way, sharp geometrical resonances, associated with diffraction, develop near the Rayleigh-Wood (RW) points, and may lead to a dramatic modification of the optical extinction and of the nearfield distribution [10-12].

In this Letter we seek to combine the cascade mechanism of local field enhancement of a nanolens with the long-range diffractive coupling encountered in periodic nanostructures. Our theoretical study is based on rigorous full-electrodynamic calculations by the layermultiple-scattering method [13,14]. The nanolens motif under consideration consists of three silver spheres with decreasing radii and separations, arranged along the $z$ axis. Following Li et al. [4] , we choose the sphere radii and separations to follow a geometric progression with ratio 2.6, although our calculations show that this choice is not critical: Other structures consisting of metallic nanoparticles with varying radii and separations can be engineered so as to provide strong EM field enhancement. The radii of the silver spheres are $R_{1}=33.8 \mathrm{~nm}$, $R_{2}=13 \mathrm{~nm}$, and $R_{3}=5 \mathrm{~nm}$, while their respective separations (surface-to-surface distances) are $d_{12}=$ $7.8 \mathrm{~nm}$ and $d_{23}=3 \mathrm{~nm}$. These parameters were chosen to fulfill a number of technical constraints: (i) Sizes and separations should be amenable to fabrication with current lithographic methods. (ii) Smaller sizes and separations would challenge the applicability of the EM model that considers local dielectric functions. (iii) In this range of sizes, the optical response of silver spheres is largely dominated by a strong dipole resonance, facilitating the physical interpretation. The nanolenses are arranged on a square periodic lattice (taken in the $x-y$ plane) with lattice constant $a$. The whole structure is embedded in a glass matrix, where the effects are more pronounced than in the case of air host. In what follows we employ the dielectric function of silver obtained by interpolating to the experimental data of Johnson and Christy [15], while for glass we take a dielectric constant equal to 2.25. A schematic view of the structure under consideration is shown in Fig. 1 .

In order to better understand the underlying physics, we start by considering a square array of the largest silver spheres $\left(R_{1}=33.8 \mathrm{~nm}\right)$, embedded in the given glass matrix, assuming incidence of $p$-polarized light at an angle 


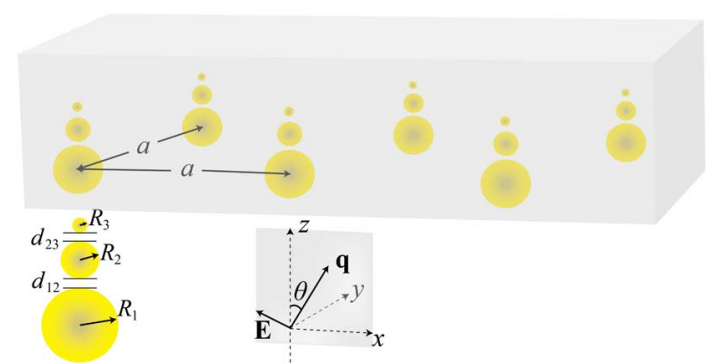

Fig. 1. (Color online) Schematic view of a square lattice of nanolenses in a glass matrix. Each nanolens consists of three silver spheres with decreasing radii $R_{1}=33.8 \mathrm{~nm}, R_{2}=13 \mathrm{~nm}$, and $R_{3}=5 \mathrm{~nm}$, and separations $d_{12}=7.8 \mathrm{~nm}$ and $d_{23}=3 \mathrm{~nm}$.

$\theta=45^{\circ}$ (the electric field lies in the $x-z$ plane). The lattice constant is taken equal to $360 \mathrm{~nm}$, a value large enough to ensure that interaction among the individual nanospheres is negligible. In this case, the extinction peaks (extinction is a dimensionless quantity, defined, as usual, as the negative natural logarithm of transmittance) shown in the upper diagram of Fig. 2 practically correspond to the plasmon modes of the isolated silver spheres. In the wavelength region under consideration, the given structure exhibits two resonances: A narrow one, at $395 \mathrm{~nm}$, due to the quadrupolar plasmon modes of the individual spheres, and a wider one, due to the dipolar plasmon modes of the spheres, which interacts with an RW anomaly at $461 \mathrm{~nm}$ and splits in two peaks. The nature of these resonances can be verified by corresponding field plots, as shown in Fig. 2 for the dipolar case. We note that, here, the first four RW anomalies appear at $922 \mathrm{~nm}$ (RW1), $522 \mathrm{~nm}$ (RW2), $461 \mathrm{~nm}$ (RW3), and $382 \mathrm{~nm}$ (RW4), and are associated with the $(-1,0)$, $(-1, \pm 1),(-2,0)$, and $(0, \pm 1)$ \& $(-2, \pm 1)$ diffraction channels, respectively.

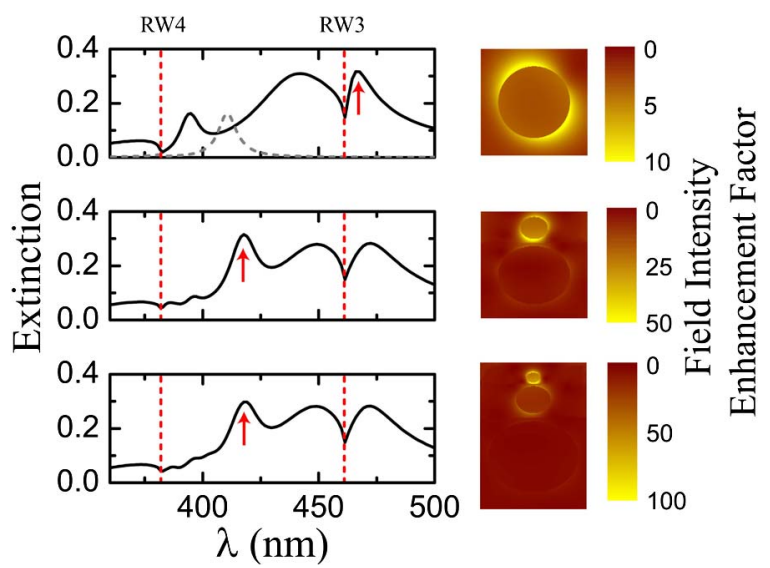

Fig. 2. (Color online) Extinction spectra of square arrays $(a=360 \mathrm{~nm})$ of silver nanospheres in a glass matrix, for $p$ polarized light incident at an angle $\theta=45^{\circ}$. Upper diagram: spheres of radius $R_{1}=33.8 \mathrm{~nm}$ (solid line) or $R_{2}=13 \mathrm{~nm}$ (dashed line). Middle diagram: nanosphere dimers $\left(R_{1}=33.8 \mathrm{~nm}, R_{2}=13 \mathrm{~nm}\right.$, and $\left.d_{12}=7.8 \mathrm{~nm}\right)$. Lower diagram: complete nanolens motif $\left(R_{1}=33.8 \mathrm{~nm}, R_{2}=13 \mathrm{~nm}\right.$, $R_{3}=5 \mathrm{~nm}, d_{12}=7.8 \mathrm{~nm}$, and $\left.d_{23}=3 \mathrm{~nm}\right)$. The vertical dashed lines mark the position of the RW anomalies RW3 and RW4. Next to the extinction diagrams we show corresponding field intensity plots at the resonance wavelengths indicated by the arrows.
We next add a second layer, consisting of the mediumsized spheres $\left(R_{2}=13 \mathrm{~nm}\right)$, arranged with the same twodimensional periodicity above the first array, so that the particles in the two layers are separated by a distance $d_{12}=7.8 \mathrm{~nm}$. The medium-sized spheres exhibit a dipolar resonance in the wavelength region of interest, about $410 \mathrm{~nm}$, as shown by dashed lines in the upper diagram of Fig. 2. When the two layers are brought in such close proximity, the plasmon modes of the individual spheres interact with each other, giving rise to a pronounced hybrid mode [16] at $417 \mathrm{~nm}$. This mode is associated with a hot spot with high EM field values about the medium sphere, as shown in the middle panel of Fig. 2.

Finally, we add a third array, consisting of the smallest spheres $\left(R_{3}=5 \mathrm{~nm}\right)$, at a distance $d_{23}=3 \mathrm{~nm}$ above the second array, so as to reproduce the original nanolens motif. The plasmon resonances of these spheres, because of their small size, are too weak, and are not discernible in the extinction spectrum of the nanolens array, which remains practically unaltered. However, as shown in the lower panel of Fig. 2, at the hot-spot resonance a clearly stronger field localization occurs now in the region between the medium and the smallest spheres, with a field intensity twice as large as in the case of the nanosphere dimer, in agreement with the results of [4]. This hot-spot hybrid plasmon mode that will concern us here is not strongly affected by changes in the lattice constant, as long as the lattice remains sparse enough and no considerable interaction with RW anomalies takes place.

We shall now examine systematically the effect of interaction between the hot-spot hybrid plasmon mode and the RW anomalies. In Fig. 3 we display extinction spectra of square arrays of the full nanolens motif as RW2 passes progressively through the hot-spot plasmon resonance. It should be stressed that the hot-spot mode does not correspond to the maximum extinction. The maximum extinction corresponds to a plasmon mode with a more or less equal field distribution in all three spheres and lower field intensity, while the hot-spot mode is associated with the small peak in the vicinity of $\lambda \approx 420 \mathrm{~nm}$, which corresponds mainly to absorption. The evolution of this resonance peak in the far-field extinction spectrum, as it crosses the RW point, exhibits the usual characteristics [10-12], namely an asymmetry in the spectral line shape and a sharp dip near the RW point. On the other hand, the interaction of the hot-spot

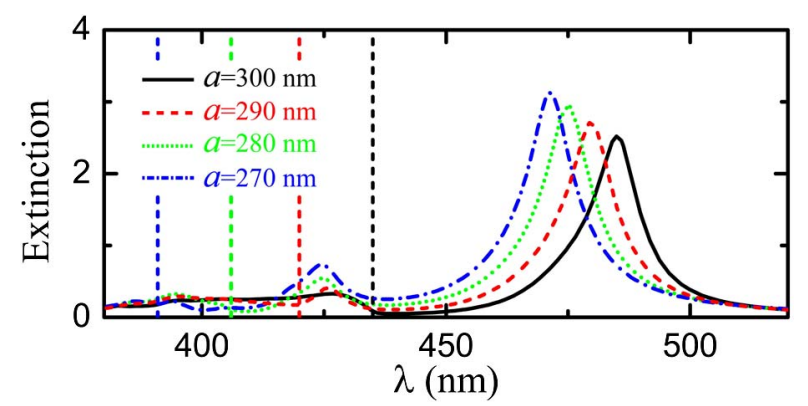

Fig. 3. (Color online) Extinction spectra of square arrays of the nanolens motif under consideration in a glass matrix, for $p$-polarized light incident at an angle $\theta=45^{\circ}$, for lattice constants $a$ varying from 270 to $300 \mathrm{~nm}$. The vertical dashed lines indicate the position of RW2 in each case. 


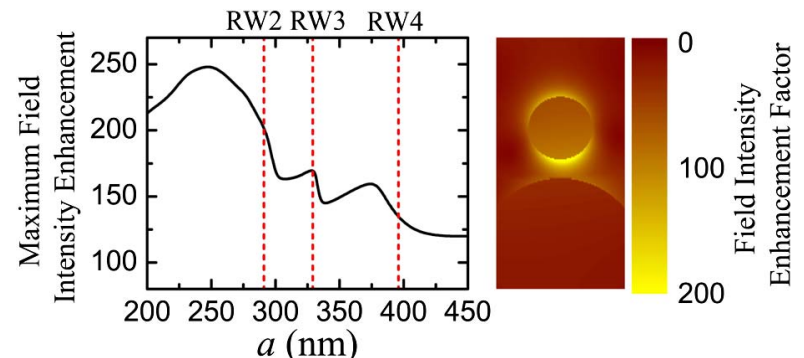

Fig. 4. (Color online) Dependence of the maximum hot-spot field intensity on the lattice constant for $p$-polarized light incident at an angle $\theta=45^{\circ}$ on square arrays of the nanolens motif under consideration in a glass matrix. The vertical dashed lines denote the lattice constants at which RW anomalies coincide with the hot-spot resonance. A field intensity plot in the region between the medium and the smallest spheres at the hot-spot resonance, for $a=250 \mathrm{~nm}$, is shown in the margin.

mode with RW anomalies is clearly manifested also in the corresponding local field enhancement.

In Fig. 4 we present the dependence of the maximum hot-spot field intensity on the lattice constant, within a lattice constant range that allows RW2, RW3, and RW4 to come into play. We note in passing that the lattice constant required for $\mathrm{RW} 1$ to become relevant $(\sim 160 \mathrm{~nm})$ is such that near-field interaction among the nanolenses is no longer negligible, leading to additional extinction peaks. The vertical dashed lines denote the lattice constants at which an RW anomaly falls at the hot-spot resonance. For smaller lattice constants, the RW anomaly is on the left side of the hot-spot resonance (shorter wavelengths), while for larger lattice constants it is on its right side. Clearly, the field intensity increases as an RW anomaly approaches the hot-spot resonance from the right and reaches its maximum when the RW point is on the left side of the resonance. In this case the diffraction edge is on the high-energy side of the hot-spot resonance and very little radiative coupling can occur, as the allowed diffracted orders are all of higher energy than the hot-spot resonance [12]. In the margin of Fig. $\underline{4}$ we show a plot of the maximum field intensity enhancement achieved by introducing diffractive coupling in the nanolens arrays. This field enhancement, about twice as large as that observed in the isolated nanolens motif, is achieved by setting the lattice constant equal to $250 \mathrm{~nm}$, at which RW2 is relevant.

In conclusion, we proposed and demonstrated with rigorous numerical simulations an original nanostructure combining two mechanisms of EM field enhancement.
On one hand, a nanolens consisting of three silver spheres with progressively decreasing sizes and separations is used to profit from a quasi-static cascade mechanism of near-field enhancement. On the other hand, multiple units of such nanolenses couple coherently in the far field through a long-range diffractive coupling characteristic of periodic arrays. The interplay between these two mechanisms reveals a rich spectral response in the near and far fields with the appearance of hybrid resonances and their interaction with RW anomalies at the diffraction edge. The parameter space of geometrical characteristics was explored to find an optimum regime of maximum field enhancement, with the two mechanisms acting in concert. This work represents a new possibility of design for hybrid nanostructures, aiming to combine the benefits of near-field and large-scale interaction between plasmonic units.

\section{References}

1. J. A. Schuller, E. S. Barnard, W. Cai, Y. C. Jun, J. S. White, and M. L. Brongersma, Nat. Mater. 9, 193 (2010).

2. P. Alonso-González, P. Albella, M. Schnell, J. Chen, F. Huth, A. García-Etxarri, F. Casanova, F. Golmar, L. Arzubiaga, L. E. Hueso, J. Aizpurua, and R. Hillenbrand, Nat. Commun. 3, 684 (2012).

3. K. D. Ko, A. Kumar, K. H. Fung, R. Ambekar, G. L. Liu, N. X. Fang, and K. C. Toussaint, Jr., Nano Lett. 11, 61 (2011).

4. K. Li, M. I. Stockman, and D. J. Bergman, Phys. Rev. Lett. 91, 227402 (2003).

5. J. Dai, F. Cajko, I. Tsukerman, and M. I. Stockman, Phys. Rev. B 77, 115419 (2008).

6. S. V. Karpov, V. S. Gerasimov, I. L. Isaev, and V. A. Markel, Phys. Rev. B 72, 205425 (2005).

7. S. Toroghi and P. G. Kik, Appl. Phys. Lett. 100, 183105 (2012).

8. B. Ding, Z. Deng, H. Yan, S. Cabrini, R. N. Zuckermann, and J. Bokor, J. Am. Chem. Soc. 132, 3248 (2010).

9. D. A. Genov, A. K. Sarychev, V. M. Shalaev, and A. Wei, Nano Lett. 4, 153 (2004).

10. S. Zou and G. C. Schatz, Chem. Phys. Lett. 403, 62 (2005).

11. V. G. Kravets, F. Schedin, and A. N. Grigorenko, Phys. Rev. Lett. 101, 087403 (2008).

12. B. Auguié and W. L. Barnes, Phys. Rev. Lett. 101, 143902 (2008).

13. N. Stefanou, V. Yannopapas, and A. Modinos, Comput. Phys. Commun. 113, 49 (1998).

14. N. Stefanou, V. Yannopapas, and A. Modinos, Comput. Phys. Commun. 132, 189 (2000).

15. P. B. Johnson and R. W. Christy, Phys. Rev. B 6, 4370 (1972).

16. P. Nordlander, C. Oubre, E. Prodan, K. Li, and M. I. Stockman, Nano Lett. 4, 899 (2004). 